J. of the Chosun Natural Science

Vol. 6, No. 3 (2013) pp. $181-182$

http://dx.doi.org/10.13160/ricns.2013.6.3.181

\title{
Remark for Certain Elliptic PDE with Exponential Nonlinearity in a Bounded Domain
}

\author{
Namkwon Kim ${ }^{\dagger}$
}

\section{Abstract}

In this note, we are concerned with a class of semi-linear elliptic pdes with exponential nonlinearity in a bounded domain. Here, the nonlinearity is more or less growing exponentially with power $p$. We consider the problem under two types of Dirichlet boundary condition. We give existence and non-existence of solutions for those problems and some asymptotics.

Key words: Semi-linear PDE, Exponential nonlinearity, Existence

\section{Introduction}

Nonlinear elliptic pdes with exponential nonlinearity arises frequently in physics and mathematics such as mean field approximations in statistical physics, static solitons in $(2+1) \mathrm{D}$ gauge models, prescirbed Gaussian curvature problems in 2 dimensional surface, etc. We here consider elliptic pdes having exponential nonlinearity in a bounded domain $\Omega$ with Dirichlet type boundary conditions. Specifically, we consider

$$
\Delta u=f_{1}\left(x, e^{u}\right)+f_{0}(x)+4 \pi \sum_{i} n_{i} \delta_{x_{i}}, \quad x \in \Omega
$$

Here, $\Omega$ is a smooth bounded domain in the two dimensional Euclidean space, $f_{1}(x, t)$ is a measurable function continuous at 0 with respoect to $t, f_{1}(x, 0)=0$, $f_{0} \in L^{q}, q>1, n_{i}$ is positive, and $x_{1} \in \Omega$.

Motivated from the Chern-Simons equations ${ }^{[1,2]}$,

$$
\Delta u=e^{2 u}-e^{u}+4 \pi \sum_{i} n_{i} \delta_{x_{i}}
$$

we restrict ourself $f_{1}$ satisfying

$$
A(x)\left(t^{p}+1\right)>f_{1}(x, t)>-A(x)
$$

for some positive $A \in L^{q}, q>1$ and $p>1$.

Department of Mathematics, Chosun University, Kwangju 501-759, Korea †Corresponding author : imnamkw@chosun.ac.kr (Received : September 2, 2013, Revised : September 16, 2013, Accepted : September 23, 2013)
Examples of such $f$ are $f(t)=t^{p}-t, t \ln t$.

We consider two types of Dirichlet boundary conditions for (1) again motivated from the Chern-Simons model;

$$
\begin{aligned}
& u \rightarrow B \text { as } x \rightarrow \partial \Omega \text { (Topological) } \\
& u \rightarrow \infty \text { as } x \rightarrow \partial \Omega \text { (Nontopological). }
\end{aligned}
$$

In this note, we shall show that (1) has a solution in the Sobolev space $H^{1}$ under the topological boundary condition and does not have a solution under the nontopological condition.

\section{Existence and Nonexistence}

\section{Theorem 1}

Let $f$ satisfy (2). Then, there exists a solution in $H^{1}$ of (1) under topological boundary condition.

proof)

We first consider a function satisfying

$$
\Delta K=f_{0}+\sum_{i} 4 \pi n_{i} \delta_{x_{i}},\left.\quad K\right|_{\partial \Omega}=B .
$$

Existence of such $K$ is guaranteed since we can decompose the above equation as follows:

$$
\begin{aligned}
& K=K_{1}+K_{2}, K_{1}=\sum_{i} n_{i} \ln \left|x-x_{i}\right|^{2}, \\
& \Delta K_{2}=f_{0},\left.\quad K_{2}\right|_{\partial \Omega}=-K_{1}+B
\end{aligned}
$$


Since $K_{1}$ is Holder continuous away from $x_{1}, K_{1}$ becomes a Holder continuous function near the boundary of $\Omega$ Hence, a Holder continuous $K_{2}$ exists by the standard elliptic theory.

It is noted that $e^{K}<C$ and $K \in L^{q}, \forall q>1$ since $K=$ $\sum_{i} n_{i} \ln \left|x-x_{i}\right|^{2}+K_{2}$.

Let $v=u-K$, then it is equivalent to show the existence of a solution of the following equation.

$$
\Delta v=f_{1}\left(x, e^{K} e^{v}\right), v=0, x \in \partial \Omega
$$

Let $f_{2}(x, t)=\frac{f_{1}(x, t)}{t}$ and $F(x, t)=\int_{1}^{t} f_{2}(x, t) d t$. The variational formulation of (3) becomes then $E(v)=$ $\frac{1}{2}\|\nabla v\|_{L^{2}}^{2}+\int_{\Omega} F\left(x, e^{K} e^{v}\right)$. The first condition of (2) implies that $F \leq \int_{1}^{e^{K} e^{v}} A\left(t^{p-1}+t^{-1}\right) \leq C A\left(e^{p(K+v)}+K+v+C\right)$. Therefore, $E$ is well defined in $H^{1}$ since $v \cdot e^{v} \in L^{q}, \forall q>1$ Next, by the second of (2),

$$
\begin{aligned}
& F\left(x, e^{K} e^{v}\right) \geq-\int_{1}^{e^{K} e^{v}} \frac{A}{t} d t \geq-A(K+v) \\
& \int_{\Omega} F \geq-\|A\|_{L^{q}}\left(\|v\|_{L^{q /(q-1)}}+\|K\|_{L^{q /(q-1)}}\right) \geq C\|\nabla v\|_{L^{2}}-C
\end{aligned}
$$

Therefore, $E$ becomes strongly coercive and a minimizing sequence converges weakly in $H^{1}$. By the first of (2) and the Trudinger embedding, $\int_{\Omega} F$ is compact in $H^{1}$ and we have a minimizer.

\section{Theorem 2.}

Let $f$ satisfy (2). Then, there does not exist a solution in $H^{1}$ of (1) under the nontopological boundary condition.

\section{proof)}

If $v$ is a solution of (1) in $H^{1}$, by standard bootstrap argument and (2), we get $v$ is Holder continuous.

Therefore, by the nontopological boundary condition, $v$ is bounded from above and thus $e^{v}$ is bounded. This in turn implies that

$$
|\Delta v|=\left|f_{1}\left(x, e^{u}\right)\right| \leq A(x)\left(1+e^{p u}\right) \leq C A \in L^{q} .
$$

Let $v=v_{1}+v_{2}, v_{1}=N * \Delta v, \Delta v_{2}=0$. Here, $N$ is the Newtonian potential. Then, $v_{1}$ is uniformly bounded and thus $v_{2}$ must be a harmonic function which tends to negative infinity near the boundary of $\Omega$ This is impossible due to the maximum principle and we finish the proof.

If we assume differentiability of $f_{1}$ near $t=0$, we can get certain compact of solutions under the topological condition. Concretely, we have the following theorem.

\section{Theorem 3.}

If the latter condition in (2) is replaced with $f_{1} \geq-\frac{A t}{1+t}$, the $H^{1}$ norm of $v$ under topological condition is uniformly bounded. And, $u \rightarrow-\infty$ uniformly as $B \rightarrow-\infty$.

\section{proof)}

Clearly, $\frac{A t}{1+t} \leq A$. Thus the proof of theorem 1, holds true. Further, (4) becomes $F\left(x, e^{K} e^{v}\right) \geq-\int_{1}^{e^{K} e^{v}} \frac{A}{1+t} d t \geq-$ $A\left(\ln \left(1+e^{K+v}\right)+1\right) \geq-A\left(v^{+}+\ln \left(2+e^{K}\right)\right)$. Here, we use the inequality $\ln \left(1+\varepsilon e^{v}\right) \leq v^{+}+\ln (1+\varepsilon)$. Then, (5) becomes $\int_{\Omega} F \geq-C\left(\left\|v \mid+\ln \left(2+e^{K}\right)\right\|_{\left.L^{q /(q-1)}\right)} \geq-C\|v\|_{L^{q /(q-1)}}-C \geq-C\|\nabla v\|_{L^{2}}-C\right.$ unoformly if $B \leq 0$. Thus, we have a uniform bound of $\|\nabla v\|_{L^{2}}$ as $B \rightarrow-\infty$.

This leads us that $u=v+K \equiv v+\tilde{K}+B$ diverges uniformly.

\section{References}

[1] J. Hong, Y. Kim, and P. Y. Pac, "Multivortex solutions of the abelian Chern-Simons-Higgs theory", Phys. Rev. Lett., Vol. 64, pp. 2230-2233, 1990.

[2] R. Jackiw and E. J. Weinberg, "Self-dual ChernSimons vortices", Phys. Rev. Lett., Vol. 64, pp. 2234-2237, 1990. 